\title{
Examination of frameworks for safe integration of intelligent small UAS into the NAS
}

\author{
Michael J. Logan, $\mathrm{PE}^{1}$ \\ NASA Langley Research Center, Hampton, VA
}

\begin{abstract}
This paper discusses a proposed framework for the safe integration of small unmanned aerial systems (sUAS) into the National Airspace System (NAS). The paper briefly examines the potential uses of sUAS to build an understanding of the location and frequency of potential future flight operations based on the future applications of the sUAS systems. The paper then examines the types of systems that would be required to meet the applicationlevel demand to determine "classes" of platforms and operations. A framework for categorization of the "intelligence" level of the UAS is postulated for purposes of NAS integration. Finally, constraints on the intelligent systems are postulated to ensure their ease of integration into the NAS.
\end{abstract}

\section{Nomenclature}

UAS = Unmanned Aircraft System

NAS $\quad=$ National Airspace System

sUAS $\quad=$ small UAS

\section{Introduction}

$\mathrm{T}$ There has been a tremendous upsurge in the desire for small, Unmanned Aerial Systems (sUAS) to be able to freely access the National Airspace System (NAS). These applications for small UAS (sUAS) vary in both their scope and potential operational impacts on the NAS. It is instructive to look at what types of applications require what types of airspace operations, what "classes" of vehicles satisfy these application demands, the salient characteristics of these small platforms, and what potential operating frameworks can be used to allow their safe operation in the NAS.

\section{Application Demand}

To determine the potential demand for sUAS system access, it is important to address both the applications for which sUAS can be used as well as the potential users of these systems and their impacts on the using communities. Broadly speaking, these applications and user communities can be broken into two main application objectives, namely, those being used for commercial purposes and those being used in the service of the "public good". (Note that there can be some overlap between applications when, for example, a regulatory requirement of a public agency is carried out by commercial firms.)

\section{A. Commercial Applications}

For the potential commercial uses of sUAS, it is easiest to target those applications for which there has already been a demand expressed. These include:

- Aerial photography and GIS

- Agriculture and crop surveys

- Outdoor advertising and banner towing

${ }^{1}$ Head, Small Unmanned Aerial Vehicle Laboratory (SUAVELab), NASA Langley Research Center, Senior Member AIAA. 
- Security services

- "Fly by" services (meter reading, aerial inventory, etc.)

- Entertainment and recreation

- Research and Development

- Fish spotting

- Training

Once airspace access has been opened to commercial users, it is likely that other applications will appear which have not been considered.

\section{B. Public Use applications}

In terms of sUAS used in the service of the "public good", there are again several applications for which there has already been an expressed demand. These public use aircraft currently must obtain a Certificate of Authorization from the FAA in order to operate as a public UAS. In terms of simple present demand, the FAA has indicated that $80 \%$ of the active CoAs for 2011 are for sUAS. The types of public operations which could be of great value include:

- Law enforcement (Federal, state, and local)

- Support to first responders (firefighters, HAZMAT)

- Traffic monitoring

- Pipeline and infrastructure monitoring (which may be conducted by commercial firms)

- Disaster response

- Weather and sever storm observation

- Volcano plume assessment

- Climate change impact monitoring (ice, snow, water, etc.)

- Environmental monitoring (air quality, runoff, etc.)

- Regulatory and taxation

- Research and Education

- Wildlife management

- Wildfire detection and support

- Department of Defense (DoD)

\section{Operational Frequency}

In terms of usage, the previous section detailed the general types of application demand. From this, it is reasonable to conclude that there are applications for sUAS in remote, rural, suburban, and urban areas. Using the application demand figures, if each potential platform averages one use per day, it is possible that sUAS operations could well exceed 200,000 per day throughout the country or over 75 million operations per year. While some operators will likely conduct numerous operations per day (law enforcement, first responders, etc.), other uses such as disaster response will likely be infrequent.

In addition, the DoD has indicated in their NAS Integration report for 2010 that there are presently 146 UAS personnel units at 63 locations throughout the US. By 2015, the DoD expects to have 197 UAS units at 105 locations needing some 275,000 flight hours per year in the NAS for training and proficiency/currency. Although this number includes all classes of UAS, the Army has indicated that of the slightly more than 1,000,000 UAS hours since 2001, over 300,000 were for sUAS. This would indicate that some 82,000 flight hours or 164,000 operations per year would be conducted by DoD using sUAS.

The FAA has indicated that of the currently active public operator CoAs issued, some $80 \%$ are for sUAS operations. This would indicate a clear bias on the part of public (Federal, state, and local) agencies to use sUAS versus their larger counterparts.

Clearly, the level of NAS access granted to sUAS will determine both the level of utilization and the impact on the NAS itself. This level of access consists not only of the geospatial constraints (where and at what altitudes) but also what equipage will be required, if any. 


\section{Small UAS Systems}

There are a myriad of small UAS platforms and systems, each designed to fulfil a particular niche in the current marketplace. It is helpful to "group" these platforms into a small set of "categories" primarily based on their concept of operations with size, weight, and performance considered as a secondary sub-category. This categorization is more consistent with the types of regulatory frameworks which may be required for operations.

In the first category, sUAS are operated as an on-demand, "point defense" system where the operator typically either hand launches the system or is a V/TOL system. These systems are typically operated with either a hand-held controller, a "smart" cell phone or tablet, or a laptop computer as its ground station. The typical application is usually within a short distance of the launch point and is within visual line of sight for the majority of its mission. Flight durations are usually on the order of 15-60 minutes. Mission ranges usually are within a 1-2 mile radius of the launch point. Flight weights typically range from under a pound to under $20 \mathrm{lbs}$. Mission altitudes are usually low to very low (200-1000ft). These systems would be most helpful to law enforcement and first responders since they are typically ad hoc, low altitude, real-time video applications.

In the second category, sUAS are operated as a stationary "patrol" system. These systems could be launched in a variety of methods including pneumatic launcher, hand or bungee launch, or runway/roadway launch. The application here is typically one of conducting a fixed route operation repeatedly. This could include both within visual line-of-sight operations and beyond line of sight operations. Typical flight durations would be from 2-24 hours, with mission ranges from 5-100 miles. These types of operations, such as pipeline monitoring, perimeter observation, or large farm crop mapping, are usually low altitude missions flown on fixed routes for long durations.

Other sUAS systems defy simple categorizations. These systems typically are special purpose units which perform a specific function. These systems can include 50-100lb. autonomous rotary wing systems for hyperspectral imaging, small fixed-wing units for high altitude cloud density measurement or in-situ storm development measurement, and others. These systems run the gamut from low altitude, short duration (15-30 minutes), visual line of sight missions to multi-hour, high altitude, satcom-equipped units. This "special purpose" class is likely to be the most difficult to seamlessly integrate into the current NAS system.

\section{Intelligence levels}

In the case of "intelligence", this poses another unique set of categorization problems, particularly as it applied to NAS integration. One way to view this paradigm is to look at the maximum level of intelligent behavior that would be "allowed" by the system. This "intelligence freedom" is different than the overall system level of intelligence as will be shown below.

At the lowest level of "intelligence" the air vehicle is essentially a remotely piloted vehicle with the pilot operating in a similar manner to traditional manned aircraft or radio control pilots. In this instance, the on-board level of intelligence is virtually non-existent while the overall "system" intelligence level is only limited by the pilot. Since the current NAS is predicated on this paradigm, there is likely to be little issue with seamlessly integrating this type of system.

In the next level, the system is typically used as an autonomous navigating vehicle where the mission profile is pre-loaded and the system autonomously flies the mission and returns. For this type of system, there is usually a two-way command and control link that provides real-time telemetry data and the ability to command the vehicle to perform basic off-mission functions such as change course, altitude, speed, etc. In this instance the maximum intelligence permitted is usually limited to adaptive inner-loop control for simple stability. Similarly, the pilot/operator is usually also limited in the flexibility of the response to extraordinary conditions.

In the "semi-smart" level, the system is somewhat adaptive in both the mission profile and the ability to respond to off-nominal conditions. An example of this would be an sUAS that is programmed to map a volcano plume or hazmat cloud. The system operator would give a general set of geographic areas to search and perhaps a search pattern method and the system would adaptively implement this. Integration of this class of system into the NAS would generally require either a larger area to scan for visual or other sense-and-avoid systems or some other method of larger area de-confliction. This would also apply to "swarming" systems in that the swarm as a whole would need to be considered as a monolithic single user in the NAS consuming a specific area of airspace.

At the "large scale intelligence" level, both the mission, location, and response to stimuli are adaptive. This presents the largest challenge to NAS integration since it is unlikely that there would exist a method to fully characterize a non-deterministic system, a requirement for the current paradigm of avionics certification. If, on the other hand, the system as a whole is treated as a manned aircraft, then it might be possible to present a small set of 
scenarios to validate that the system performs in accordance with normal procedures. In short, a test and "check ride" for the system similar to the way manned aircraft pilots are currently tested. This would effectively separate the hardware reliability function check, which can usually be measured and/or analyzed directly, and the adaptive algorithmic side of the system which cannot. How this could be implemented effectively has yet to be determined.

\section{SUAS and the NAS}

\section{A. Regulatory Framework}

The current rules for NAS access comprise both "airworthiness" (e.g. 14 CFR 23, 25 for aircraft) as well as "operations" (e.g. 14 CFR 91 and others). However, in considering what rules may or may not be applicable to sUAS it is instructive to keep in mind that the current regulations are predicated on two basic premises: First, that aircraft should be made safe for their occupants, and Second, that aircraft should be operated so as to keep them from colliding with one another to prevent harm to both sets of occupants. With sUAS, there are no occupants. Therefore the primary focus of much of the highly detailed proscriptive nature of many of the regulations is irrelevant or not applicable to sUAS.

However, taken as a whole, the current regulations can provide some insight into a "level of risk" that is already assumed by current NAS users that could be utilized as a potential framework for sUAS inclusion. Another potential issue not generally addressed by current regulations is the potential risk to ground personnel and property. This would clearly be more important to sUAS since many of the applications being considered involve low altitude operations in and around urban areas.

One consideration that often creates exceptional debate is the question of "How 'small' is small?". Rather than attempt to create a definition directly, it might be possible to create some categories of "small" based upon certain risk levels already in the current regulations.

First, it is reasonable to postulate that there exists a size of sUAS that is so small that it presents almost no potential risk or hazard to either other aircraft or ground personnel no matter where it operates or who operates it. There is presently no data that would definitively identify the upper limit of what this size, weight, and/or kinetic energy might be. Testing sUAS of different types colliding with aircraft components, ground "targets" and instrumented anthropomorphic dummies would provide this limit.

The next potential category involves looking at existing regulations for potential insight on current collision risk hazard abatement. The existing Part 23 regulation calls for a bird-strike windshield protection requirement of $2 \mathrm{lb}$. The existing Part 25 regulations call for bird strike resistance of $4 \mathrm{lb}$. to the windshield, 8lb. to the engine and airframe. Further, Part 101 defines a "free balloon" and indicates:

"(4) Except as provided for in §101.7, any unmanned free balloon that-

(i) Carries a payload package that weighs more than four pounds and has a weight/size ratio of more than three ounces per square inch on any surface of the package, determined by dividing the total weight in ounces of the payload package by the area in square inches of its smallest surface;

(ii) Carries a payload package that weighs more than six pounds;

(iii) Carries a payload, of two or more packages, that weighs more than 12 pounds; or

(iv) Uses a rope or other device for suspension of the payload that requires an impact force of more than 50 pounds to separate the suspended payload from the balloon.”

These existing rules define a "level of hazard" that is associated with birds and free balloons in terms of a collision hazard that is currently an "assumed risk" for users of the NAS. It is possible to use similar verbiage to construct a sUAS rule that would allow sUAS to be similar in weight and density to the existing free balloon payloads, i.e., 12lb. total, no single component more than 6lb. and no component more dense than 3oz./in^ $\wedge$. This would provide an adequate size for a large variety of small UAS operations described in earlier sections.

Part 101 also proscribes certain limits on the locations from which free balloons may operate and other operational limitations. However, many of these operational limitations are due to the lack of maneuverability and control of the balloon's trajectory, which would not be an issue with sUAS. If such characteristics were taken into consideration, it is likely that this size sUAS could operate in large classes of airspace without equipage or other constraints as they would pose no more of a hazard than free balloon payloads. In addition, since birds typically are unpredictable, they represent more of a hazard to current NAS users than sUAS operations as sUAS operations would be controlled either autonomously or via human operator. 
The FAA issued an Advisory Circular, AC-91-57, that has been used as the basis for operations of many "model aircraft" operations since 1981. This voluntary compliance document suggests that model aircraft operations restrict their altitudes to $400 \mathrm{ft}$. or less, and if within 3 miles of an airport, to notify the airport operator or cognizant ATC facility. Note that AC 91-57 does not have any size limitation specifically called for. Many current model aircraft operations regularly exceed 400ft. AGL and many model aircraft clubs operate at airports/airfields and have done so safely for decades. One of the most prominent model aircraft organizations, the Academy of Model Aeronautics (AMA), has a member safety code. This code calls for models to generally be 55lb. or less, although a "waiver" process exists to allow models up to 100lb., to be flown at AMA sanctioned airfields and events. Furthermore, the AMA also has an active "turbine waiver" program that provides turbine powered model aircraft, some of which can fly up to 200kts., to also operate under AMA rules and insurance coverage. However, there is presently no published data that indicates that operating under such a safety code can be directly attributed to any specific increase in safety nor is there any data to support a lack of such a safety code would cause a reduction in net safety. Furthermore, there is a general lack of data concerning the reliability of COTS systems and subsystems that could be used in a safety hazard risk analysis. This includes COTS autopilot reliability and navigational accuracy, command and control link reliability, particularly in urban areas when using unlicensed bands, servoactuator reliability, motor and battery reliability, video link stability, ground station reliability, many of which are Windows based computers, systemlevel reliability, and others.

All present model, sUAS hobbyists, and "toy" aircraft flyers yield right-of-way to manned aircraft and suspend operations when manned aircraft are in the vicinity. This presents a rulemaking dilemma. Generally, in the manned aircraft rules, slower, less maneuverable aircraft have the right of way over faster. Thus, gliders must yield to balloons, powered aircraft must yield to gliders, faster aircraft must yield to slower, etc. The sUAS interaction with manned aircraft whereby sUAS yield to manned aircraft is a convention not due to a specific rule requirement (although it is suggested in AC 91-57) but reflects the reality that it is far easier for a ground based operator to see and avoid a large manned aircraft than it is for a manned aircraft to see and avoid a sUAS. Specific experiments need to be conducted to determine the ability of manned aircraft pilots to detect and discern the intent of sUAS under a variety of conditions. Only when such ability is quantified can a rule and its impact on other NAS users' ability to see-and-avoid be assessed to any significant degree. This is particularly true when the "avoid" part of the rule may differ from existing convention, such as would be the case with sUAS yielding to manned aircraft.

Attempting to discern an "upper limit" on what defines a "small" UAS is also difficult. Current NAS users include Part 103 Ultralight operations. For these operations, empty weight is generally limited to 254lb. With a pilot and fuel, this represents a vehicle of some 500lb. in weight that can be travelling up to 55kt. airspeed. Note that there are relatively few restrictions on where ultralight aircraft may operate since they may operate in virtually any airspace class "with permission" and are specifically excluded from many of the rules constraining Part 91 operations. Typically, these operations are conducted during daylight (or civil twilight if equipped with anticollision lighting) and may not be operated over ill-defined "congested areas". In addition, there are no requirements for airworthiness, registration, or pilot aeronautical knowledge. Clearly, this represents the "upper limit" of the current risk/hazard in terms of vehicle size and kinetic energy. It also helps define at least one boundary, by exclusion, of what size potentially non-airworthy vehicle should NOT be allowed to fly directly over people (since flight over open-air assemblies are prohibited under Part 103). Note also that for experimental certificated aircraft, there are relaxed restrictions once a certain number of hours of successful flight have been demonstrated by the aircraft. It is reasonable to consider that some form of "proof time" might be appropriate for larger sUAS that wish to fly over populated areas. As a "reinforcement" to this upper limit possibility, some foreign aviation authorities are considering 150kg. (330lb.) as an upper limit to what is considered "small". It is clear that an arbitrary limitation for what defines the upper limit of small is neither desirable nor defensible.

\section{Operational Framework}

It is conceivable that there would be varying levels of access for sUAS based upon credible risk assessments and mitigations of those risks. For example, a property owner should be allowed to operate a "small" UAS over their own property up to an altitude of 500ft. This would be consistent with allowing the operator, such as a small farm, to assume an "acceptable" risk if they so choose. Also, since manned aircraft are constrained by regulation to maintain a minimum altitude above people and structures, there would be very little likelyhood of collision with other NAS users. Similarly, in urban areas, small UAS could operate at or below the surrounding building heights (or up to 250ft. above - similar to tethered balloons) without likelihood that a manned aircraft would be present. Model aircraft and hobbyist operations would continue as they are currently conducted with the same suggested $400 \mathrm{ft}$. operating altitude (with higher altitudes allowed under controlled conditions). Operators of sUAS under these 
circumstances would not be required to undergo the same medical and testing that manned aircraft pilots currently do.

For other operations, risk could be mitigated by a variety of means. Primarily, those sUAS whose size and weight make them inherently safe should be allowed unrestricted access to the NAS. As the size and kinetic energy increases, there would need to be a corresponding increase in the control reliability to ensure that the vehicle is under positive control at all times (either performing its mission, performing a flight termination, or navigating directly to a pre-determined safe rally point). Exactly what these risk levels are and what risk mitigations can be applied needs to be the subject of a vigorous research effort.

Equippage would also need some investigation. For most operations of sUAS, there would be little benefit to the requirement for equipment such as ADS-B or a sense-and-avoid system. However, in the case of using sUAS for higher altitude operations, such equipage may be necessary to enter certain classes of airspace.

In addition, the "categorization" above may be helpful in determining a possible course of action. For those systems operating at altitudes below the current manned aircraft minimum altitudes, typically 500ft. AGL, there may be little regulation necessary for visual (or even beyond) line of sight because the risk of collision with manned aircraft is low. Furthermore, it is possible that with fixed routes such as pipelines, etc., that sectional charts could include cautions about low-level sUAS operations similar to the way electrical power lines and towers are noted as "obstructions".

For operations at airfields, current procedures may well be adequate to allow routine operations for sUAS. This would depend greatly on the type of launch and recovery method of the sUAS, however. In addition, unlike most other types of sUAS operations, it is likely that some level of "pilot" training might be required to ensure sufficient knowledge of typical ATC procedures for airfield operations.

\section{Conclusions}

Small UAS have a broad base of both commercial and public use applications. If relatively unrestricted access to the NAS is granted, the potential economic impacts could be enormous. Further, it is likely that many sUAS operations can be conducted in a safe and effective manner with no regulation. Other operations should only be regulated to the level of risk they pose to other NAS users and ground personnel. 